\title{
A Direct Discontinuous Galerkin Method with Interface Correction for the Compressible Navier-Stokes Equations on Unstructured Grids
}

\author{
Jian Cheng*, Huiqiang Yue, Shengjiao Yu and Tiegang Liu \\ School of Mathematics and Systems Science, Beihang University, Beijing 100091, China
}

Received 27 February 2017; Accepted (in revised version) 24 April 2017

\begin{abstract}
Since the original DDG method has been introduced by Liu et al. [8] in 2009, a variety of DDG type methods have been proposed and further developed. In this paper, we further investigate and develop a new DDG method with interface correction (DDG (IC)) as the discretization of viscous and heat fluxes for the compressible Navier-Stokes equations on unstructured grids. Compared to the original DDG method, the newly developed DDG (IC) method demonstrates its superior in delivering the optimal order of accuracy under demanding situations. Strategies in extension and application of this newly developed DDG (IC) method for solving the compressible Navier-Stokes equations and special treatments designed for handling boundary viscous fluxes are presented and examined in this work. The performance of the new DDG method with interface correction is carefully evaluated and assessed through a number of typical test cases. Numerical experiments show that the new DDG method with interface correction can achieve the optimal order of accuracy on both uniform structured grids and nonuniform unstructured grids, which clearly indicates its potential for further applications of real engineering practices.
\end{abstract}

AMS subject classifications: 65M60, 76N15

Key words: Direct discontinuous Galerkin method, high-order method, compressible NavierStokes equations.

\section{Introduction}

Discontinuous Galerkin (DG) methods [1-3,16,18], as a typical representative in the community of high order methods, have been widely used in computational fluid dynamics, computational acoustics, and computational magneto-hydrodynamics. The DG methods combine two advantageous features commonly associated with finite element (FE)

*Corresponding author.

Email: chengjian@buaa.edu.cn (J. Cheng) 
and finite volume methods (FVM). As in classical FE methods, accuracy is obtained by means of high-order polynomial approximation within an element rather than by wide stencils as in the case of FVM. Similar to FVM, the physics of wave propagation is, however, accounted for by solving the Riemann problems that arise from the discontinuous representation of the solution at element interfaces.

DG methods are indeed a natural choice for solving hyperbolic equations, such as the compressible Euler equations. However, the DG formulation is far less certain and advantageous for diffusion equations such as the compressible Navier-Stokes equations, where viscous and heat fluxes exist and require the evaluation of the solution derivatives at the interfaces. Taking a simple arithmetic mean of the solution derivatives from the left and right is inconsistent, because it does not take into account a possible jump of the solutions. Thus, how to appropriately address this issue becomes one of the core challenges for the practice of DG methods for real applications.

The first attempt at using DGM to solve elliptic and parabolic problems can be tracked back to the late 1970s and early 1980s when an interior penalty (IP) method was independently proposed and studied in [20,21]. In the IP method, a viscous flux is obtained through the average of the left and right state and then penalizing with a penalty term which includes the jump of the solution at each cell interface. The IP method with different stabilization terms was further analyzed for the two dimensional compressible Navier-Stokes equations by Hartmann et al. $[22,23]$, which led to a new symmetric IP (SIP) method. Inspired by the great success of the DGM for a first-order system, a natural choice to solve a second-order system is to convert it into a first-order system by introducing additional variables, and then to apply a DG method directly to the first-order system. Based on different choices of numerical flux at the cell interface, there are mainly two kinds of approaches: one is the first Bassi-Rebay (BR1) scheme [18], and the other is the so-called local discontinuous Galerkin (LDG) method [15]. A variation of a LDG method, termed compact DG (CDG) method [17], was later developed by Peraire and Persson to overcome the issue that LDG method is not compact when applied to multidimensional problems. For the same reason, Bassi and Rebay introduced the second Bassi and Rebay scheme (BR2) based on BR1 scheme in order to maintain the compactness and stability for the pure diffusion problems [14]. In practice, the auxiliary variables in both BR2 scheme and CDG method are usually eliminated by introducing the so-called local and global lifting operator. From the finite volume community, van Leer et al. $[6,7]$ proposed a recovery-based DG (RDG) method for diffusion equations using the recovery principle, that recovers a smooth continuous solution that in the weak sense is indistinguishable from the discontinuous discrete solution. Similarly, Luo et al. $[4,5]$ developed a reconstructed DG (rDG) method for the compressible Navier-Stokes equations on arbitrary grids, where a smooth continuous solution is reconstructed at each cell interface from the discontinuous discrete solution and the diffusive fluxes are then obtained based on the smooth reconstructed solution. Besides, a kind of hybridizable DG (HDG) discretization was recently introduced for the solution of convection-diffusion equations [24]. For an uniform analysis and comparison among several of the methods 
above, one can refer to the paper [19].

From another perspective, based on the direct weak formulation of DG framework and the analysis of solution structure for pure diffusion equations, Gassner et al. [27] presented a method named the diffusive generalized Riemann problem (dGRP) method. Later, a direct discontinuous Galerkin (DDG) method was introduced by Liu and Yan [8] to construct the numerical flux for diffusion problems. Compared to the IP method, the DDG method can be viewed as a multi-term penalty method. The numerical flux defined by the DDG method is simple, compact, conservative, and consistent. The most remarkable feature of the DDG method is its simplicity in implementation and its efficiency in computational cost. Very recently, the DDG method has been successfully extended and applied for solving the more complex compressible Navier-Stokes equations $[25,26]$ on arbitrary grids. The DDG method shows its potential to deliver comparable accuracy as the widely used BR2 method at a significantly reduced computational cost, thus, becomes an attractive alternative to discretize the compressible Navier-Stokes equations on arbitrary grids.

Since the original DDG method has first been introduced in 2009, a variety of DDG type methods have been proposed and further developed to fix the potential difficulties in the practical implementations or to enhance the optimal order of accuracy on nonuniform grids. In order to avoid that the jump terms of higher order $(k \geq 4)$ derivatives in the original DDG discretization, a new DDG method with interface correction was introduced and further analyzed by Liu et al. $[9,10]$ for solving convection diffusion problems. Meanwhile, due to the reason that the formulation of the original DDG method lacks symmetric properties, which could make the traditional $L^{2}$ error analysis become difficult, a symmetric DDG method is developed by Yan and his coworkers [12,13]. The newly developed DDG type methods mentioned above possessed the merit and beauty of the original DDG method, meanwhile offer a tantalizing glimpse of further investigation and evaluation of their full potential for more complex and real applications.

In this paper, we further investigate and develop the new DDG method with interface correction (DDG (IC)) as the discretization of viscous and heat fluxes in the compressible Navier-Stokes equations on unstructured grids. The performance of the new DDG method with interface correction is carefully evaluated and assessed for solving the compressible Navier-Stokes equations for practical problems. Strategies in extension and application this newly developed DDG formulation for the compressible Navier-Stokes have been detailed described and examined. Special boundary treatment is designed to address the issue that the first and second derivatives required in the DDG flux functions are generally unknown at the ghost states of the boundary. A number of test cases are presented to assess the performance the DDG method with interface correction for solving the compressible Navier-Stokes equations. Numerical experiments demonstrate that the new DDG method with interface correction can achieve the optimal order of accuracy on both uniform and nonuniform unstructured grids, which clearly indicates its potential for further application in real engineering practices.

The rest of the paper is organized as follows. The governing equations are briefly de- 
scribed in Section 2. The extension and implementation of the DDG method with interface correction for discretizing the viscous and heat fluxes in the Navier-Stokes equations are presented and discussed in Section 3. Numerical experiments are reported in Section 4. Concluding remarks are given in Section 5.

\section{Governing equations}

Let $\Omega$ be the computational domain in $\mathbb{R}^{2}$, the two dimensional steady-state compressible Navier-Stokes equations are given by:

$$
\nabla \cdot \mathcal{F}(\boldsymbol{u})-\nabla \cdot \mathcal{G}(\boldsymbol{u}, \nabla \boldsymbol{u})=0 \quad \text { in } \Omega,
$$

where $\boldsymbol{u}=(\rho, \rho u, \rho v, E)^{\mathrm{T}}$ is the solution vector consisting of the density $\rho$, velocity vector $\boldsymbol{v}=(u, v)^{\mathrm{T}}$, and the specific total energy $E$. The convective flux functions $\mathcal{F}(\boldsymbol{u})$ are defined as

$$
\mathcal{F}(\boldsymbol{u})=\left(\mathcal{F}_{x}(\boldsymbol{u}), \mathcal{F}_{y}(\boldsymbol{u})\right)=\left(\begin{array}{cc}
\rho u & \rho v \\
\rho u^{2}+p & \rho u v \\
\rho u v & \rho v^{2}+p \\
(E+p) u & (E+p) v
\end{array}\right)
$$

Here, $p$ is pressure which is determined by the equation of state of an ideal gas, i.e.,

$$
p=(\gamma-1)\left(E-\frac{1}{2} \rho\left(u^{2}+v^{2}\right)\right)
$$

where $\gamma=1.4$ is the heat capacity ratio for air.

The viscous flux functions $\mathcal{G}(\boldsymbol{u}, \nabla \boldsymbol{u})$ are defined as

$$
\begin{aligned}
\mathcal{G}(\boldsymbol{u}, \nabla \boldsymbol{u}) & =\left(\mathcal{G}_{x}(\boldsymbol{u}, \nabla \boldsymbol{u}), \mathcal{G}_{y}(\boldsymbol{u}, \nabla \boldsymbol{u})\right) \\
& =\left(\begin{array}{cc}
0 & 0 \\
\tau_{x x} & \tau_{x y} \\
\tau_{y x} & \tau_{y y} \\
u \tau_{x x}+v \tau_{x y}+\mathcal{K} T_{x} & u \tau_{y x}+v \tau_{y y}+\mathcal{K} T_{y}
\end{array}\right),
\end{aligned}
$$

where $\mathcal{K}$ is the thermal conductivity coefficient. The viscous stress tensor can be computed from

$$
\tau=\mu\left(\nabla v+(\nabla v)^{T}-\frac{2}{3}(\nabla \cdot \boldsymbol{v}) \mathrm{I}\right) .
$$

Finally, the temperature $T$ is defined by

$$
\mathcal{K} T=\frac{\mu \gamma}{\operatorname{Pr}} \frac{E-\frac{1}{2} \rho v^{2}}{\rho} .
$$


In the above formulas, $\mathrm{Pr}$ is the Prandtl number, which is taken as 0.72 for sea-level air, and $\mu$ is the dynamic viscosity coefficient which can be computed by Sutherland's law as follows:

$$
\frac{\mu}{\mu_{0}}=\left(\frac{T}{T_{0}}\right)^{\frac{3}{2}} \frac{T_{0}+S}{T+S}
$$

where $\mu_{0}$ and $T_{0}$ denote the reference dynamic viscosity coefficient and reference temperature respectively, and $S$ is assumed as a constant with a value of $110 \mathrm{~K}$.

In order to derive the proposed DDG spatial discretization, we rewrite the viscous flux functions $\mathcal{G}(\boldsymbol{u}, \nabla \boldsymbol{u})$ as follows:

$$
\begin{aligned}
\mathcal{G}(\boldsymbol{u}, \nabla \boldsymbol{u}) & =\left(\mathcal{G}_{x}(\boldsymbol{u}, \nabla \boldsymbol{u}), \mathcal{G}_{y}(\boldsymbol{u}, \nabla \boldsymbol{u})\right) \\
& =\left(\left(\mathcal{G}_{x x} \mathcal{G}_{x y}\right) \cdot \nabla \boldsymbol{u},\left(\mathcal{G}_{y x} \mathcal{G}_{y y}\right) \cdot \nabla \boldsymbol{u}\right),
\end{aligned}
$$

where

$$
\begin{aligned}
& \mathcal{G}_{x x}=\frac{\partial \mathcal{G}_{x}}{\partial \boldsymbol{u}_{x}} \\
& =\frac{\mu}{\rho}\left(\begin{array}{cccc}
0 & 0 & 0 & 0 \\
-\frac{4}{3} u & \frac{4}{3} & 0 & 0 \\
-v & 0 & 1 & 0 \\
-\left(\frac{4}{3} u^{2}+v^{2}+\frac{\gamma}{P r}\left(\frac{E}{\rho}-v^{2}\right)\right) & \left(\frac{4}{3}-\frac{\gamma}{P r}\right) u & \left(1-\frac{\gamma}{P r}\right) v & \frac{\gamma}{P r}
\end{array}\right), \\
& \mathcal{G}_{x y}=\frac{\partial \mathcal{G}_{x}}{\partial u_{y}}=\frac{\mu}{\rho}\left(\begin{array}{cccc}
0 & 0 & 0 & 0 \\
\frac{2}{3} v & 0 & -\frac{2}{3} & 0 \\
-u & 1 & 0 & 0 \\
-\frac{1}{3} u v & v & -\frac{2}{3} u & 0
\end{array}\right) \text {, } \\
& \mathcal{G}_{y x}=\frac{\partial \mathcal{G}_{y}}{\partial u_{x}}=\frac{\mu}{\rho}\left(\begin{array}{cccc}
0 & 0 & 0 & 0 \\
-v & 0 & 1 & 0 \\
\frac{2}{3} u & -\frac{2}{3} & 0 & 0 \\
-\frac{1}{3} u v & -\frac{2}{3} v & u & 0
\end{array}\right) \text {, } \\
& \mathcal{G}_{y y}=\frac{\partial \mathcal{G}_{y}}{\partial \boldsymbol{u}_{y}} \\
& =\frac{\mu}{\rho}\left(\begin{array}{cccc}
0 & 0 & 0 & 0 \\
-u & 1 & 0 & 0 \\
-\frac{4}{3} v & 0 & \frac{4}{3} & 0 \\
-\left(u^{2}+\frac{4}{3} v^{2}+\frac{\gamma}{P r}\left(\frac{E}{\rho}-v^{2}\right)\right) & \left(1-\frac{\gamma}{P r}\right) u & \left(\frac{4}{3}-\frac{\gamma}{P r}\right) v & \frac{\gamma}{P r}
\end{array}\right) .
\end{aligned}
$$


For the sake of simplification, we introduce the following definition:

$$
\boldsymbol{G}(\boldsymbol{u}) \nabla \boldsymbol{u}:=\left(\left(\mathcal{G}_{x x} \mathcal{G}_{x y}\right) \cdot \nabla \boldsymbol{u},\left(\mathcal{G}_{y x} \mathcal{G}_{y y}\right) \cdot \nabla \boldsymbol{u}\right) .
$$

Finally, we obtain the equivalent form of the primal compressible Navier-Stokes equations in Eq. (2.1) as follows:

$$
\nabla \cdot \mathcal{F}(\boldsymbol{u})-\nabla \cdot(\boldsymbol{G}(\boldsymbol{u}) \nabla \boldsymbol{u})=0 \quad \text { in } \Omega
$$

\section{Direct DG method with interface correction for the compressible Navier-Stokes equations}

\subsection{Review of original Direct DG method}

Let us begin this section with introducing some basic notations. The shape-regular subdivision of domain $\Omega$ is denoted by $\mathcal{T}_{h}=\{\kappa\}$. For $\kappa \in \mathcal{T}_{h}$, we denote the outward unit normal of $\partial \kappa$ by $\boldsymbol{n}_{\kappa}:=\left(n_{x}, n_{y}\right)^{\mathrm{T}}$ and we use $\boldsymbol{u}^{+}$and $\boldsymbol{u}^{-}$to denote the traces of $\boldsymbol{u}$ evaluated from the interior and the exterior of $\partial \kappa$, respectively. Then, we define the average and jump of $\boldsymbol{u}$ on $\partial \kappa$ as follows:

$$
\overline{\boldsymbol{u}}=\frac{\boldsymbol{u}^{-}+\boldsymbol{u}^{+}}{2}, \quad[\boldsymbol{u}]=\boldsymbol{u}^{-}-\boldsymbol{u}^{+} .
$$

For matrices $\boldsymbol{a}, \boldsymbol{b} \in \mathbb{R}^{m \times n}$, we define the matrix multiplication operator $\boldsymbol{a}: \boldsymbol{b}=\sum_{i=1}^{m} \sum_{j=1}^{n} \boldsymbol{a}_{i j} \boldsymbol{b}_{i j}$ and for vectors $\boldsymbol{c} \in \mathbb{R}^{m}, \boldsymbol{d} \in \mathbb{R}^{n}$ the dyadic tensor $\boldsymbol{c} \otimes \boldsymbol{d} \in \mathbb{R}^{m \times n}$ is defined by $(\boldsymbol{c} \otimes \boldsymbol{d})_{i j}=c_{i} d_{j}$. Additionally, we introduce the following broken Sobolev space

$$
\mathcal{V}_{h, p}=\left\{\boldsymbol{u} \in\left[L_{2}(\Omega)\right]^{4}:\left.\boldsymbol{u}\right|_{\kappa} \in\left[\mathcal{P}^{p}\right]^{4}, \forall \kappa \in \mathcal{T}_{h}\right\},
$$

where $\mathcal{P}^{p}$ denotes the polynomial of degree $p$. For $\kappa \in \mathcal{T}_{h}, \partial \kappa \cap \partial \Omega \neq \varnothing$ is the boundary face, we denote the set of all boundary faces by $\Gamma$. An interior edge of $\mathcal{T}_{h}$ is the onedimensional interior of $\partial \kappa^{+} \cap \partial \mathcal{\kappa}^{-}$, where $\kappa^{+}$and $\kappa^{-}$are two adjacent elements of $\mathcal{T}_{h}$.

In order to obtain the DG discretization of Eq. (2.10), we multiply them by a test function $v_{h} \in \mathcal{V}_{h, p}$ and integrate by parts over element $k$, then, we get the following weak formulation: find $\boldsymbol{u}_{h} \in \mathcal{V}_{h, p}$, such that

$$
\begin{aligned}
& -\int_{\mathcal{K}} \mathcal{F}\left(\boldsymbol{u}_{h}\right): \nabla \boldsymbol{v}_{h} d \boldsymbol{x}+\int_{\partial \mathcal{K}}\left(\mathcal{F}\left(\boldsymbol{u}_{h}\right) \cdot \boldsymbol{n}_{\mathcal{K}}\right) \cdot \boldsymbol{v}_{h} d s \\
& \quad+\int_{\mathcal{K}}\left(\boldsymbol{G}\left(\boldsymbol{u}_{h}\right) \nabla \boldsymbol{u}_{h}\right): \nabla \boldsymbol{v}_{h} d \boldsymbol{x}-\int_{\partial \kappa}\left(\boldsymbol{G}\left(\boldsymbol{u}_{h}\right) \nabla \boldsymbol{u}_{h}\right) \cdot \boldsymbol{n}_{\mathcal{K}} \cdot \boldsymbol{v}_{h} d s=0, \quad \forall \boldsymbol{v}_{h} \in \mathcal{V}_{h, p} .
\end{aligned}
$$

Summing over all the elements $\kappa \in \mathcal{T}_{h}$ and approximating the inviscid and viscous flux functions at each cell interface and boundary face by suitable numerical flux functions, 
we obtain the following DDG discretization: find $\boldsymbol{u}_{h} \in \mathcal{V}_{h, p}$, such that

$$
\begin{aligned}
& -\int_{\Omega} \mathcal{F}\left(\boldsymbol{u}_{h}\right): \nabla \boldsymbol{v}_{h} d \boldsymbol{x}+\sum_{\kappa \in \mathcal{T}_{h}} \int_{\partial \kappa} \mathcal{H}\left(\boldsymbol{u}_{h}^{+}, \boldsymbol{u}_{h}^{-}, \boldsymbol{n}_{\kappa}\right) \cdot \boldsymbol{v}_{h}^{+} d s \\
& \quad+\int_{\Omega}\left(\boldsymbol{G}\left(\boldsymbol{u}_{h}\right) \nabla \boldsymbol{u}_{h}\right): \nabla \boldsymbol{v}_{h} d \boldsymbol{x}-\sum_{\kappa \in \mathcal{T}_{h}} \int_{\partial \kappa}\left(\widehat{\boldsymbol{G}} \widehat{\left(\boldsymbol{u}_{h}\right) \nabla} \boldsymbol{u}_{h}\right) \cdot \boldsymbol{n}_{\kappa} \cdot \boldsymbol{v}_{h}^{+} d s=0, \quad \forall \boldsymbol{v}_{h} \in \mathcal{V}_{h, p}
\end{aligned}
$$

where $\mathcal{H}(\cdot, \cdot, \cdot)$ is the inviscid numerical flux function, such as Local Lax-Friedrichs, Roe and HLLC flux, etc. Unlike BR2 and SIP method, here, in the DDG discretization, the viscous numerical flux functions are constructed based on the jump of conservative variables themselves and their even order derivatives directly based on DG weak form.

Namely, let us take DG (P2) discretization as an example, in that case all the even order derivatives higher than the second order derivatives are zero, thus, omitted. Then, the DDG flux for Eq. (3.4) can be written as follows:

$$
\begin{aligned}
& \widehat{\boldsymbol{G}} \widehat{\left(\boldsymbol{u}_{h}\right) \nabla} \boldsymbol{u}_{h}=\underbrace{\overline{\boldsymbol{G}\left(\boldsymbol{u}_{h}\right)}\left(\frac{\beta_{0}}{\tilde{h}}\left[\boldsymbol{u}_{h}\right] \otimes \boldsymbol{n}_{\kappa}\right)}_{\text {jump of solution }}+\underbrace{\overline{\boldsymbol{G}\left(\boldsymbol{u}_{h}\right) \nabla \boldsymbol{u}_{h}}}_{\text {average of viscous term }} \\
& +\beta_{1} \tilde{h} \underbrace{\left(\left[\left(\boldsymbol{G}\left(\boldsymbol{u}_{h}\right)\left(\nabla \boldsymbol{u}_{h}\right)_{x}\right) \cdot \boldsymbol{n}_{\kappa}\right]\left[\left(\boldsymbol{G}\left(\boldsymbol{u}_{h}\right)\left(\nabla \boldsymbol{u}_{h}\right)_{y}\right) \cdot \boldsymbol{n}_{\kappa}\right]\right)}_{\text {jump of second order derivatives }},
\end{aligned}
$$

where

$$
\begin{aligned}
& {\left[\left(\boldsymbol{G}\left(\boldsymbol{u}_{h}\right)\left(\nabla \boldsymbol{u}_{h}\right)_{x}\right) \cdot \boldsymbol{n}\right]:=\left[\mathcal{G}_{x x} \boldsymbol{u}_{x x}+\mathcal{G}_{x y} \boldsymbol{u}_{y x}\right] n_{x}+\left[\mathcal{G}_{y x} \boldsymbol{u}_{x x}+\mathcal{G}_{y y} \boldsymbol{u}_{y x}\right] n_{y}} \\
& {\left[\left(\boldsymbol{G}\left(\boldsymbol{u}_{h}\right)\left(\nabla \boldsymbol{u}_{h}\right)_{y}\right) \cdot \boldsymbol{n}\right]:=\left[\mathcal{G}_{x x} \boldsymbol{u}_{x y}+\mathcal{G}_{x y} \boldsymbol{u}_{y y}\right] n_{x}+\left[\mathcal{G}_{y x} \boldsymbol{u}_{x y}+\mathcal{G}_{y y} \boldsymbol{u}_{y y}\right] n_{y}}
\end{aligned}
$$

Note that in the above formulas, for brevity, we omit the subscript $h$ of $\boldsymbol{u}$.

The choice of the parameters $\left(\beta_{0}, \beta_{1}\right)$ is based on both the strict analysis of the convergence and super-convergence property of the DDG method for the convection diffusion equations, cf. $[10,11]$ and our numerical experiments for solving practical problems of the compressible Navier-Stokes equations. We remark that the original theoretical analysis is based on the scalar convection diffusion equation, though such a requirement is also likely to be necessary for more complex Navier-Stokes equations. However, based on our limited numerical experiences, it seems that the $\beta_{0}$ needs to be chosen large enough for the consideration of stability in some cases. The numerical experiments indicate that, roughly, choose the $\beta_{0}$ as the same scale of $k^{2}$ for $P_{k}$ polynomials $(k \geq 2)$ will be good enough to stabilize the scheme meanwhile without bringing any detrimental effects to the order of accuracy. In specific, in this work, we simply choose $\beta_{0}$ equal to $2,4,9$ for $k=1,2,3$, respectively and for the second parameter $\beta_{1}$, we set it equal to $1 / 12$ in all our simulations.

The characteristic length $\tilde{h}$ can be easily defined as the cell size when the numerical flux is implemented on a uniform structure grid. However, on non-uniform meshes, such 


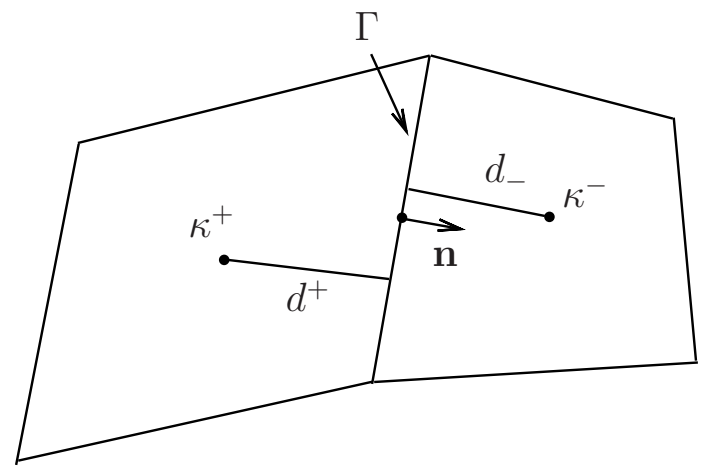

(a) For internal face

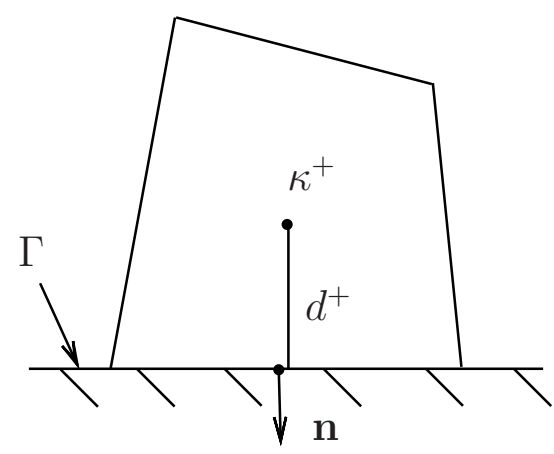

(b) For boundary face

Figure 1: Definition of characteristic length $\tilde{h}$ for the DDG flux.

as unstructured grids or highly anisotropic grids which is commonly used in the region of boundary layers, the appropriate definition of characteristic length $\tilde{h}$ can play a decisive role on both the magnitude of error and the order of accuracy for the DDG type methods. Generally, the basic principle in defining the characteristic length $\tilde{h}$ on arbitrary grids is that the characteristic length $\tilde{h}$ should be associated with the target interface where the viscous flux is evaluated and also be orthogonal to the common interface or boundary face. A variety of different definitions had been tested in our previous work [25], and the one gives the best performance is given as follows:

$$
\tilde{h}:= \begin{cases}d^{+}+d^{-} & \text {for internal face } \Gamma, \\ d^{+} & \text {for boundary face } \Gamma,\end{cases}
$$

where $d^{+}$and $d^{-}$are the distance from the cell center to the target face as shown in Fig. 1, similarly, for boundary faces, $\tilde{h}$ is defined as the distance from cell center to boundary face. It should be noted that this definition is also works quite well when high-order curved grids are used for handling curved geometries. In that case, the distance $d^{+}$and $d^{-}$will be obtained by calculating the distance from cell center to the curved interface or boundary face, approximately.

\subsection{Direct DG method with interface correction}

Furthermore, in order to obtain the new direct DG discretization with interface correction (DDG (IC)) for the compressible Navier-Stokes equations, an additional term which contains the numerical flux of test function needs to be added to Eq. (3.4), then, the new 
discretization is given as: find $\boldsymbol{u}_{h} \in \mathcal{V}_{h, p}$, such that

$$
\begin{aligned}
& -\int_{\Omega} \mathcal{F}\left(\boldsymbol{u}_{h}\right): \nabla \boldsymbol{v}_{h} d \boldsymbol{x}+\sum_{\kappa \in \mathcal{T}_{h}} \int_{\partial \kappa} \mathcal{H}\left(\boldsymbol{u}_{h}^{+}, \boldsymbol{u}_{h}^{-}, \boldsymbol{n}_{\kappa}\right) \cdot \boldsymbol{v}_{h}^{+} d s \\
& \quad+\int_{\Omega}\left(\boldsymbol{G}\left(\boldsymbol{u}_{h}\right) \nabla \boldsymbol{u}_{h}\right): \nabla \boldsymbol{v}_{h} d \boldsymbol{x}-\sum_{\kappa \in \mathcal{T}_{h}} \int_{\partial \kappa}\left(\widehat{\boldsymbol{G}} \widehat{\left(\boldsymbol{u}_{h}\right) \nabla} \boldsymbol{u}_{h}\right) \cdot \boldsymbol{n}_{\kappa} \cdot \boldsymbol{v}_{h}^{+} d s \\
& \quad+\sum_{\kappa \in \mathcal{T}_{h}} \int_{\partial \kappa}\left[\boldsymbol{u}_{h}\right] \cdot\left(\left(\boldsymbol{G}^{\widehat{\mathrm{T}}\left(\boldsymbol{u}_{h}\right) \nabla} \boldsymbol{v}_{h}\right) \cdot \boldsymbol{n}_{\kappa}\right) d s=0, \quad \forall \boldsymbol{v}_{h} \in \mathcal{V}_{h, p}
\end{aligned}
$$

where $\widehat{\boldsymbol{G}^{\mathrm{T}}} \widehat{\left(\boldsymbol{u}_{h}\right) \nabla} \boldsymbol{v}_{h}$ can be given as follows:

$$
\widehat{\boldsymbol{G}^{\mathrm{T}}} \widehat{\left(\boldsymbol{u}_{h}\right) \nabla} \boldsymbol{v}_{h}=\overline{\boldsymbol{G}^{\mathrm{T}}\left(\boldsymbol{u}_{h}\right) \nabla \boldsymbol{v}_{h}}
$$

and $\boldsymbol{G}^{\mathrm{T}}\left(\boldsymbol{u}_{h}\right) \nabla \boldsymbol{v}_{h}$ is defined by

$$
\boldsymbol{G}^{\mathrm{T}}\left(\boldsymbol{u}_{h}\right) \nabla \boldsymbol{v}_{h}:=\left(\left(\mathcal{G}_{x x}^{\mathrm{T}} \mathcal{G}_{y x}^{\mathrm{T}}\right) \cdot \nabla \boldsymbol{v}_{h},\left(\mathcal{G}_{x y}^{\mathrm{T}} \mathcal{G}_{y y}^{\mathrm{T}}\right) \cdot \nabla \boldsymbol{v}_{h}\right)
$$

Compared to the original DDG discretization described in Section 3.1, the newly developed DDG method with interface correction enjoys several superior potentials. Firstly, as the previous work [9] indicates, the newly developed DDG method with interface correction no longer requires the jump terms of even order derivatives higher than two. Only the jump of second order derivatives is required for all the DG (Pk) discretization, which greatly simplifies the implementation of this method for very high-order practices. Secondly, the newly developed DDG method becomes more robust in delivering the designed optimal order of accuracy under demanding situations, i.e., under severely distorted grids, which will be demonstrate later through our numerical experiments.

In the numerical fluxes of test function $v_{h}$ given above, the value of test functions and their derivatives are equal to zero outside the element $\kappa$, therefore, only inside of $\kappa$ contributes to $\boldsymbol{G}^{\mathrm{T}} \widehat{\left(\boldsymbol{u}_{h}\right) \nabla} \boldsymbol{v}_{h}$, i.e.,

$$
\boldsymbol{G}^{\mathrm{T}} \widehat{\left(\boldsymbol{u}_{h}\right) \nabla} \boldsymbol{v}_{h}=\frac{\boldsymbol{G}^{\mathrm{T}}\left(\boldsymbol{u}_{h}^{+}\right) \nabla \boldsymbol{v}_{h}^{+}}{2} .
$$

Furthermore, splitting the faces into interior faces and boundary faces, we can rewrite the new DDG discretization with interface correction as follows: find $\boldsymbol{u}_{h} \in \mathcal{V}_{h, p}$, such that

$$
\begin{aligned}
& -\int_{\Omega} \mathcal{F}\left(\boldsymbol{u}_{h}\right): \nabla \boldsymbol{v}_{h} d \boldsymbol{x}+\sum_{\kappa \in \mathcal{T}_{h}} \int_{\partial \kappa \backslash \Gamma} \mathcal{H}\left(\boldsymbol{u}_{h}^{+}, \boldsymbol{u}_{h}^{-}, \boldsymbol{n}_{\kappa}\right) \cdot \boldsymbol{v}_{h}^{+} d s \\
& +\int_{\Omega}\left(\boldsymbol{G}\left(\boldsymbol{u}_{h}\right) \nabla \boldsymbol{u}_{h}\right): \nabla \boldsymbol{v}_{h} d \boldsymbol{x}-\sum_{\kappa \in \mathcal{T}_{h}} \int_{\partial \kappa \backslash \Gamma}\left(\widehat{\boldsymbol{G}} \widehat{\left(\boldsymbol{u}_{h}\right) \nabla} \boldsymbol{u}_{h}\right) \cdot \boldsymbol{n}_{\kappa} \cdot \boldsymbol{v}_{h}^{+} d s \\
& +\sum_{\kappa \in \mathcal{T}_{h}} \int_{\partial \kappa \backslash \Gamma}\left[\boldsymbol{u}_{h}\right] \cdot\left(\left(\boldsymbol{G}^{\widehat{\mathrm{T}}\left(\boldsymbol{u}_{h}\right) \nabla} \boldsymbol{v}_{h}\right) \cdot \boldsymbol{n}_{\kappa}\right) d s \\
& +\int_{\Gamma} \mathcal{H}\left(\boldsymbol{u}_{h}^{+}, \boldsymbol{u}_{\Gamma}\left(\boldsymbol{u}_{h}^{+}\right), \boldsymbol{n}_{\kappa}\right) d s-\int_{\Gamma}\left(\boldsymbol{G}\left(\widehat{\boldsymbol{u}_{\Gamma}} \widehat{\left(\boldsymbol{u}_{h}^{+}\right)}\right) \nabla \boldsymbol{u}_{h}\right) \cdot \boldsymbol{n}_{\kappa} \cdot \boldsymbol{v}_{h}^{+} d s
\end{aligned}
$$




$$
+\int_{\Gamma}\left(\boldsymbol{u}_{\Gamma}\left(\boldsymbol{u}_{h}^{+}\right)-\boldsymbol{u}_{h}^{+}\right) \cdot\left(\left(\boldsymbol{G}^{\mathrm{T}}\left(\widehat{\boldsymbol{u}_{\Gamma}\left(\boldsymbol{u}_{h}^{+}\right)}\right) \nabla \boldsymbol{v}_{h}\right) \cdot \boldsymbol{n}_{\kappa}\right) d s=0, \quad \forall \boldsymbol{v}_{h} \in \mathcal{V}_{h, p}
$$

where the boundary function $\boldsymbol{u}_{\Gamma}\left(\boldsymbol{u}_{h}^{+}\right)$is given according to the type of boundary condition imposed.

Remark 3.1. We point out that the above spatial discretization of DDG method with interface correction is, to some extend, very similar to the SIP method. In fact, for the DG (P1) discretization, the above spatial discretization degenerates to the SIP discretization, though it becomes different for DG (Pk) with $k \geq 2$ as the jump of second-order derivatives must be taken into account in the DDG type methods. However, what we want to emphasize is that the major difference between the DDG type methods and the SIP method comes from the concept and derivation rather than from the specific numerical implementation. Compared to the SIP method which the derivation is based on the application of internal penalty ideas on the numerical flux for the decoupled first-order systems derived from the Navier-Stokes equations, the formulation of DDG type methods is directly based on the weak formulation of DG method and an appropriate approximation of generalized Riemann problem from pure diffusive equation. Although, these two type of methods eventually lead to the similar formulation in spatial discretization from different perspectives.

Remark 3.2. Another potential advantage of the new DDG method with interface correction, compared to the original DDG method, for solving the compressible Navier-Stokes equations is that the new DDG (IC) method is adjoint consistent. Although, adjoint consistency may not bring too much influence to the order of accuracy in solving primal problems, it can become crucial in the context of adjoint-based error estimation and adaptivity [30]. As adjoint inconsistent discretization can not deliver the optimal rates of convergence.

\subsection{Boundary treatment}

Note that the numerical flux functions defined in Eq. (3.5) and Eq. (3.9) involve the derivatives of conservative variables at ghost states of the boundary faces. In general, these derivatives are not available for practical problems. Therefore on boundary faces, we drop the jump terms of second order derivatives and only use the internal first order derivatives to define the viscous numerical flux functions, i.e.,

$$
\boldsymbol{G}\left(\widehat{\boldsymbol{u}_{\Gamma}} \widehat{\left(\boldsymbol{u}_{h}^{+}\right)}\right) \nabla \boldsymbol{u}_{h}=\boldsymbol{G}\left(\boldsymbol{u}_{\Gamma}\left(\boldsymbol{u}_{h}^{+}\right)\right)\left(\frac{\beta_{0}}{\tilde{h}}\left(\boldsymbol{u}_{\Gamma}\left(\boldsymbol{u}_{h}^{+}\right)-\boldsymbol{u}_{h}^{+}\right) \otimes \boldsymbol{n}_{\mathcal{\kappa}}\right)+\boldsymbol{G}\left(\boldsymbol{u}_{\Gamma}\left(\boldsymbol{u}_{h}^{+}\right)\right) \nabla \boldsymbol{u}_{h}^{+},
$$

and

$$
\boldsymbol{G}^{\mathrm{T}}\left(\widehat{\boldsymbol{u}_{\Gamma}\left(\widehat{\boldsymbol{u}_{h}^{+}}\right)}\right) \nabla \boldsymbol{v}_{h}=\boldsymbol{G}^{\mathrm{T}}\left(\boldsymbol{u}_{\Gamma}\left(\boldsymbol{u}_{h}^{+}\right)\right) \nabla \boldsymbol{v}_{h}^{+}
$$


where $\boldsymbol{u}_{\Gamma}\left(\boldsymbol{u}_{h}^{+}\right)$is boundary function defined based on the specific type of boundary conditions. For example along no-slip adiabatic wall boundaries, the no-slip boundary condition enforces the velocity equal to zero, thus

$$
\boldsymbol{u}_{\Gamma}\left(\boldsymbol{u}_{h}^{+}\right)=\left(\rho^{+}, 0,0, E^{+}\right)^{\mathrm{T}} .
$$

Furthermore, on adiabatic wall boundaries, $\nabla T \cdot \boldsymbol{n}=0$, therefore the viscous flux functions $\mathcal{G}(\boldsymbol{u}, \nabla \boldsymbol{u})$ can be defined as

$$
\mathcal{G}^{\text {adia }}(\boldsymbol{u}, \nabla \boldsymbol{u})=\left(\begin{array}{cc}
0 & 0 \\
\tau_{x x} & \tau_{x y} \\
\tau_{y x} & \tau_{y y} \\
0 & 0
\end{array}\right) .
$$

For more details about boundary treatment for the compressible Navier-Stokes equation$\mathrm{s}$, we refer the readers to [22] and references cited therein.

\subsection{Pseudo-transient continuation}

We add a pseudo time term to Eq. (2.10), i.e., and rewrite the steady-state Navier-Stokes equations as

$$
\frac{\partial \boldsymbol{u}}{\partial t}+\nabla \cdot \mathcal{F}(\boldsymbol{u})-\nabla \cdot(\boldsymbol{G}(\boldsymbol{u}) \nabla \boldsymbol{u})=0 \quad \text { in } \Omega
$$

Spatial discretization of Eq. (3.17) leads to a system of ordinary differential equations:

$$
\mathcal{M} \frac{d \boldsymbol{u}_{h}}{d t}=\mathcal{R}\left(\boldsymbol{u}_{h}\right)
$$

where $\mathcal{M}$ is mass matrix and $\mathcal{R}$ is right hand side residual. In this work, as we are interested in stationary problems, thus, the implicit backward Euler scheme is applied. After temporal discretization and linearizing Eq. (3.18) in time, we get a linear system written as

$$
\left(\frac{\mathcal{M}}{\Delta t} \mathrm{I}-\left.\frac{\partial \mathcal{R}}{\partial \boldsymbol{u}}\right|_{\boldsymbol{u}_{h}^{n}}\right) \Delta \boldsymbol{u}_{h}^{n}=\mathcal{R}\left(\boldsymbol{u}_{h}^{n}\right)
$$

where $\Delta t$ is time increment, and $\Delta \boldsymbol{u}_{h}^{n}=\boldsymbol{u}_{h}^{n+1}-\boldsymbol{u}_{h}^{n}$. Then, the linear system is solved by GMRES method with ILU0 preconditioning in this work.

\section{Numerical examples}

\subsection{A model problem with a source term}

In order to investigate the accuracy and convergence of the proposed DDG (IC) method for solving the compressible Navier-Stokes equations, in the first numerical test, a model problem with a source term is chosen. The exact solution to Eq. (2.1) is chosen to be

$$
\mathbf{u}=\left(\sin (2(x+y)+4), \sin (2(x+y)+4), \sin (2(x+y)+4),(\sin (2(x+y)+4))^{2}\right)^{\mathrm{T}}
$$


with a source term $\mathbf{s}$ given as follows

$$
\mathbf{s}=\left(\begin{array}{c}
4 \cos (2(x+y)) \\
\cos (2(x+y))(14 \gamma-10)+\sin (4(x+y))(2 \gamma-2) \\
\cos (2(x+y))(14 \gamma-10)+\sin (4(x+y))(2 \gamma-2) \\
\cos (2(x+y))(28 \gamma+4)+4 \gamma \sin (4(x+y))+\frac{8 \mu \gamma}{\operatorname{Pr}} \sin (2(x+y))
\end{array}\right) .
$$

Additionally, the computational domain is chosen to be $\Omega=[0,1]^{2}$ and the dynamic viscosity coefficient $\mu=0.1$.

In this test case, the Dirichlet boundary condition is enforced in the ghost states along the boundary, namely,

$$
\left.\mathbf{u}\right|_{\partial \Omega}=\left(\sin (2(x+y)+4), \sin (2(x+y)+4), \sin (2(x+y)+4),(\sin (2(x+y)+4))^{2}\right)^{\mathrm{T}} .
$$

It should be noted that although, in this test case, the first and second order derivatives can be handily derived, we still strictly follow the boundary treatment given in Section 3.3 and do not use them in the formulation of boundary viscous flux.

The initial condition has also been carefully chosen in this test case, instead of using the so-called freestream constant values, the initial condition is set as follows:

$$
\mathbf{u}_{0}=\left(\begin{array}{c}
\sin (1.5(x+y))+3 \\
0.2 \sin (1.5(x+y))+3 \\
0.8 \sin (1.5(x+y))+3 \\
(\sin (1.5(x+y))+3)^{2}
\end{array}\right)
$$

Three different types of grids are designed to assess the performance of the new DDG method with interface correction in this test case. As shown in Fig. 2, uniform structured grids, nonuniform unstructured grids together with a specially designed heterogeneous grids are used in this test case and grid convergence studies based on the three different types of grids are performed. It should be pointed out that the heterogeneous grids are specially designed, inspired by the work [31], as this type grids can be really effective in testing the performance of the viscous discretization under the demanding situations.

The $L_{2}$ norm of the solution vector $\left\|\boldsymbol{u}-\boldsymbol{u}_{h}\right\|_{L_{2}(\Omega)}$ is considered as the measure of the magnitude of the error in this test case. A comparison between the original DDG method and the newly developed DDG (IC) method is performed and presented for DG (Pk), $k=1,2,3$ and numerical results of this accuracy test are presented in Table 1, Table 2 and Table 3.

From these numerical results we note that, on both the uniform structured grids and the nonuniform unstructured grids, both the original DDG method and the newly developed DDG (IC) method can deliver the optimal order of accuracy for all the DG (Pk) methods with $k=1,2,3$, respectively. Meanwhile, the magnitude of errors for both of these methods are totally comparable. As the situation on heterogeneous grids shown 


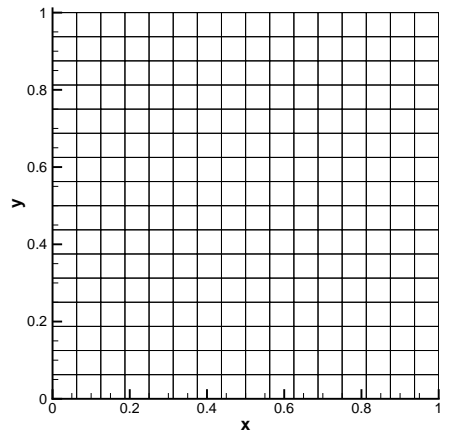

(a) Structured grid, 256

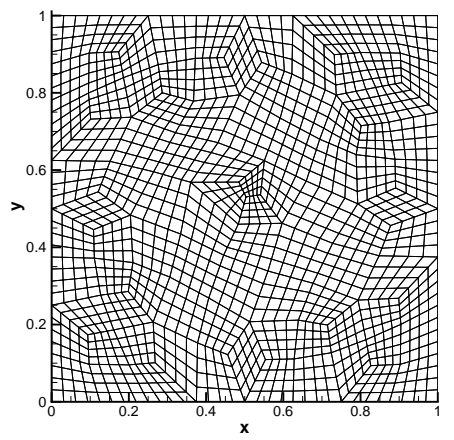

(b) Unstructured grid, 1456

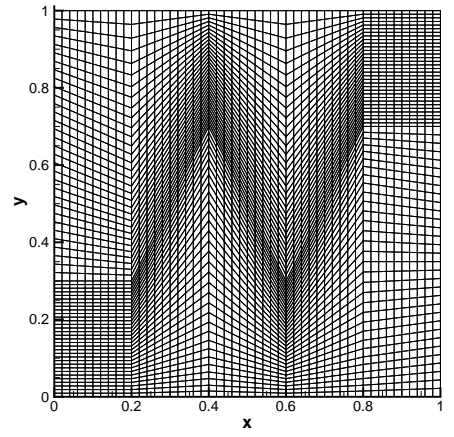

(c) Heterogeneous grid, 3200 quadrilateral cells, 289 nodes quadrilateral cells, 1521 nodes quadrilateral cells, 3315 nodes

Figure 2: Three different types of quadrilateral grids used for model problem with source term.

in Table 3, for the odd order of DG discretization, i.e., DG (Pk) $k=1,3$, both of these two methods gain designed order of accuracy with matchable errors. However, for the even order DG discretization $k=2$, it is clearly that DDG (IC) method shows its robustness over the original DDG method and gives a better performance in delivering the optimal third order of accuracy, while the original DDG method degenerates to slight higher than second order of accuracy, eventually. The grid convergence study shows that the newly developed DDG method can achieve its designed order of accuracy and has its own superior potential and robustness in handling demanding situations.

Table 1: Grid convergence study on uniform structured grids.

\begin{tabular}{||c|c|c|c|c||}
\hline Number of DoFs & DDG (P1) & Order & DDG (IC) (P1) & Order \\
\hline 48 & $2.154 \mathrm{e}-1$ & & $2.437 \mathrm{e}-1$ & \\
\hline 192 & $5.382 \mathrm{e}-2$ & 2.00 & $6.565 \mathrm{e}-2$ & 1.89 \\
\hline 768 & $1.307 \mathrm{e}-2$ & 2.04 & $1.649 \mathrm{e}-2$ & 1.99 \\
\hline 3071 & $3.195 \mathrm{e}-3$ & 2.03 & $4.097 \mathrm{e}-3$ & 2.01 \\
\hline Number of DoFs & DDG (P2) & Order & DDG (IC) (P2) & Order \\
\hline 96 & $1.687 \mathrm{e}-2$ & & $1.756 \mathrm{e}-2$ & \\
\hline 384 & $2.089 \mathrm{e}-3$ & 3.01 & $2.106 \mathrm{e}-3$ & 3.05 \\
\hline 1536 & $2.604 \mathrm{e}-4$ & 3.00 & $2.589 \mathrm{e}-4$ & 3.02 \\
\hline 6144 & $3.246 \mathrm{e}-5$ & 3.00 & $3.238 \mathrm{e}-5$ & 2.99 \\
\hline Number of DoFs & DDG (P3) & Order & DDG (IC) (P3) & Order \\
\hline 160 & $1.523 \mathrm{e}-3$ & & $1.548 \mathrm{e}-3$ & \\
\hline 640 & $9.571 \mathrm{e}-5$ & 3.99 & $9.605 \mathrm{e}-5$ & 4.01 \\
\hline 2560 & $6.003 \mathrm{e}-6$ & 4.00 & $5.992 \mathrm{e}-6$ & 4.00 \\
\hline 10240 & $3.767 \mathrm{e}-7$ & 3.99 & $3.756 \mathrm{e}-7$ & 4.00 \\
\hline \multicolumn{4}{|l}{} &
\end{tabular}


Table 2: Grid convergence study on nonuniform unstructured grids.

\begin{tabular}{||c|c|c|c|c||}
\hline Number of DoFs & DDG (P1) & Order & DDG (IC) (P1) & Order \\
\hline 273 & $7.206 \mathrm{e}-2$ & & $7.284 \mathrm{e}-2$ & \\
\hline 1092 & $1.820 \mathrm{e}-2$ & 1.98 & $1.861 \mathrm{e}-2$ & 1.97 \\
\hline 4368 & $4.424 \mathrm{e}-3$ & 2.04 & $4.629 \mathrm{e}-3$ & 2.01 \\
\hline Number of DoFs & DDG (P2) & Order & DDG (IC) (P2) & Order \\
\hline 546 & $4.719 \mathrm{e}-3$ & & $4.714 \mathrm{e}-3$ & \\
\hline 2184 & $5.565 \mathrm{e}-4$ & 3.08 & $5.538 \mathrm{e}-4$ & 3.08 \\
\hline 8736 & $6.745 \mathrm{e}-5$ & 3.04 & $6.596 \mathrm{e}-5$ & 3.07 \\
\hline Number of DoFs & DDG (P3) & Order & DDG (IC) $(\mathrm{P} 3)$ & Order \\
\hline 910 & $3.160 \mathrm{e}-4$ & & $3.169 \mathrm{e}-4$ & \\
\hline 3640 & $1.994 \mathrm{e}-5$ & 3.99 & $1.975 \mathrm{e}-5$ & 4.04 \\
\hline 14560 & $1.222 \mathrm{e}-6$ & 4.03 & $1.207 \mathrm{e}-6$ & 4.03 \\
\hline
\end{tabular}

Table 3: Grid convergence study on heterogeneous grids.

\begin{tabular}{||c|c|c|c|c||}
\hline Number of DoFs & DDG (P1) & Order & DDG (IC) (P1) & Order \\
\hline 2400 & $1.672 \mathrm{e}-2$ & & $1.768 \mathrm{e}-2$ & \\
\hline 9600 & $3.992 \mathrm{e}-3$ & 2.07 & $4.237 \mathrm{e}-3$ & 2.06 \\
\hline 38400 & $9.667 \mathrm{e}-4$ & 2.05 & $1.019 \mathrm{e}-3$ & 2.06 \\
\hline Number of DoFs & DDG (P2) & Order & DDG (IC) (P2) & Order \\
\hline 4800 & $3.568 \mathrm{e}-4$ & & $3.204 \mathrm{e}-4$ & \\
\hline 19200 & $5.288 \mathrm{e}-5$ & 2.75 & $3.860 \mathrm{e}-5$ & 3.05 \\
\hline 76800 & $1.033 \mathrm{e}-5$ & 2.36 & $4.802 \mathrm{e}-6$ & 3.01 \\
\hline Number of DoFs & DDG (P3) & Order & DDG (IC) (P3) & Order \\
\hline 8000 & $9.402 \mathrm{e}-6$ & & $8.959 \mathrm{e}-6$ & \\
\hline 32000 & $5.596 \mathrm{e}-7$ & 4.07 & $5.324 \mathrm{e}-7$ & 4.07 \\
\hline 128000 & $3.424 \mathrm{e}-8$ & 4.03 & $3.254 \mathrm{e}-8$ & 4.03 \\
\hline
\end{tabular}

\subsection{Couette flow}

In the second numerical test, a compressible Couette flow on a rectangle domain $(0 \leq$ $x \leq 2 H, 0 \leq y \leq H)$ is considered. This example is again aimed at verifying the accuracy and convergence of the DDG (IC) method for solving the compressible Navier-Stokes equations based on different types of grids.

The exact solution to this problem is given by

$$
\begin{aligned}
& u=\bar{y} U, \quad v=0, \\
& p=p_{\infty}, \quad \rho=\frac{p}{R T}, \\
& T=T_{0}+\bar{y}\left(T_{1}-T_{0}\right)+\bar{y}(1-\bar{y}) \frac{\operatorname{Pr} U^{2}}{2 c_{p}},
\end{aligned}
$$

where $\bar{y}=\frac{y}{H}, c_{p}$ is the specific heat capacity at constant pressure, $U$ is the velocity at 


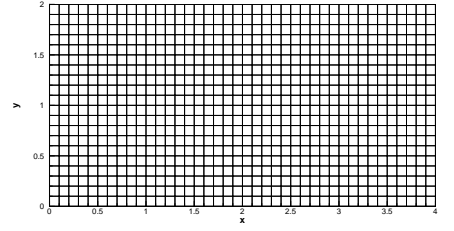

(a) Structured mesh, 800

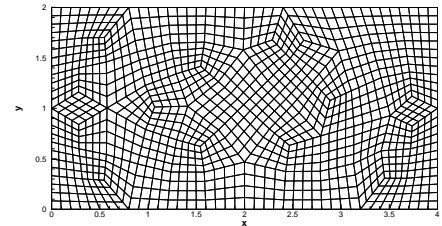

(b) Unstructured mesh, 1152

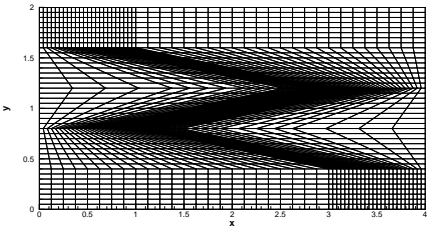

(c) Irregular mesh, 1920 quadrilateral cells, 861 nodes quadrilateral cells, 1217 nodes quadrilateral cells, 2009 nodes

Figure 3: Three different types of quadrilateral grids used for Couette flow.

upper wall and $T_{0}, T_{1}$ are the temperature at bottom and upper wall respectively. In this test case, we take $H=2.0, T_{0}=0.8$, and $T_{1}=0.85$. Mach number for upper wall is given as $M a=0.1$ and the dynamic viscosity coefficient is taken as a constant $\mu=0.1$.

Similarly, three different types of grids are adopted to assess the performance of the newly developed DDG (IC) method, as shown in Fig. 3, including the uniform structured grids, nonuniform unstructured grids together with the heterogeneous grids. A grid convergence study based on the three different types of grids is performed and the $L_{2}$ norm of density $\rho$ is used as the measure of the magnitude of the error in this study. Again in the boundary treatment of this test case, we strictly follow the boundary treatment described in Section 3.3 and do not use the first or second order derivatives at the ghost states along the boundary faces.

Numerical results are shown in Table 4. Except in some extreme situations that the magnitude of error becomes too small and contaminated, the new DDG method with interface correction provides satisfactory results. It can be seen clearly that optimal order

Table 4: Grid convergence study of DDG (IC) method for Couette flow.

\begin{tabular}{||c|c|c|c|c|c|c||}
\hline & \multicolumn{7}{|c||}{ Uniform grid } \\
\hline Number of cells & DG (P1) & Order & DG (P2) & Order & DG (P3) & Order \\
\hline 50 & $2.749 \mathrm{e}-05$ & & $1.216 \mathrm{e}-07$ & & $4.398 \mathrm{e}-10$ & \\
\hline 200 & $6.669 \mathrm{e}-06$ & 2.04 & $1.508 \mathrm{e}-08$ & 3.01 & $3.062 \mathrm{e}-11$ & 3.85 \\
\hline 800 & $1.686 \mathrm{e}-06$ & 1.98 & $1.989 \mathrm{e}-09$ & 2.92 & & - \\
\hline & \multicolumn{7}{|c||}{ Unstructured grid } \\
\hline Number of cells & DG (P1) & Order & DG (P2) & Order & DG (P3) & Order \\
\hline 72 & $6.103 \mathrm{e}-04$ & & $3.524 \mathrm{e}-06$ & & $2.315 \mathrm{e}-07$ & \\
\hline 288 & $1.866 \mathrm{e}-04$ & 1.71 & $4.000 \mathrm{e}-07$ & 3.13 & $1.451 \mathrm{e}-08$ & 4.00 \\
\hline 1152 & $4.253 \mathrm{e}-05$ & 2.13 & $4.592 \mathrm{e}-08$ & 3.12 & $9.676 \mathrm{e}-10$ & 3.91 \\
\hline & \multicolumn{7}{|c||}{ Heterogeneous grid } \\
\hline Number of cells & DG (P1) & Order & DG (P2) & Order & DG (P3) & Order \\
\hline 120 & $8.211 \mathrm{e}-06$ & \multicolumn{7}{|c|}{$1.532 \mathrm{e}-08$} & & $3.268 \mathrm{e}-11$ & \\
\hline 480 & $2.210 \mathrm{e}-06$ & 1.89 & $2.031 \mathrm{e}-09$ & 2.91 & & - \\
\hline 1920 & $6.182 \mathrm{e}-07$ & 1.83 & $2.650 \mathrm{e}-10$ & 2.93 & - & - \\
\hline
\end{tabular}


of accuracy has been achieved on all those three different types of grids including the heterogeneous grids which can be regarded as quite challenging.

\subsection{Laminar flow over a NACA0012 airfoil}

This test case involves a subsonic laminar flow past a NACA0012 airfoil at a Mach number of 0.5 , an angle of attack of $0^{\circ}$, and a Reynolds number of 5,000 based on the freestream velocity and the chord length of the airfoil. An adiabatic wall boundary is assumed along the airfoil in this test case. The Reynolds number is close to the upper limit of a steady flow. This computation is performed on a hybrid structured and unstructured quadrilateral mesh with 3,466 elements as shown in Fig. 4(a). A distinguishing feature of this test case is the separation of the flow occurring near the tailing edge, which causes the formation of two small recirculation bubbles in the wake region. This can be clearly seen from the streamline plot in the vicinity of the trailing edge as shown in Fig. 4(b). Fig. 5 shows the computed skin friction coefficients and pressure coefficients obtained by the DDG (IC) (P2) and DDG (IC) (P3) methods, which match very well compared to the reference values. A detailed comparison of the computed drag coefficients due to pressure $\left(C d_{p}\right)$ and viscous stress $\left(C d_{f}\right)$, respectively, is shown in Table 5 . The residual convergence history shown in Fig. 6 . Due to the reason that the grid is designed to be relatively coarse, we can see that the higher-order DDG (IC) method, especially DDG (IC) (P3) method, matches the reference values very well. The numerical results indicate that the newly developed DDG (IC) method has the capability to deliver satisfactory results for this typical simulation.

Table 5: Comparison of pressure coefficient and drag coefficient for laminar flow over a NACA0012 airfoil.

\begin{tabular}{||c|c|c|c||}
\hline Method & $C d_{p}$ & $C d_{f}$ & $C d$ \\
\hline DDG (IC) (P1) & 0.011698 & 0.040698 & 0.052397 \\
DDG (IC) (P2) & 0.020653 & 0.035096 & 0.055749 \\
DDG (IC) (P3) & 0.022445 & 0.032228 & 0.054673 \\
\hline References [22,25,29] & $0.02208-0.02250$ & $0.03103-0.03283$ & $0.05311-0.05533$ \\
\hline
\end{tabular}

\subsection{Steady flow past a cylinder}

A subsonic flow past a circular cylinder at a Reynolds number of 40 based on the diameter of the cylinder and at a Mach number of 0.2 is considered in this test case. Steady and unsteady laminar flow behind a circular cylinder, representing flow around bluff bodies, has been subjected to numerous experimental and computational studies. The flow considered in this test case is steady and characterized by the presence of a symmetric pair of closed separation bubbles at the current Reynolds number. The initial condition is a uniform freestream with no-slip adiabatic boundary condition on the solid wall. The hybrid structured and unstructured quadrilateral mesh used in the computation and shown in 


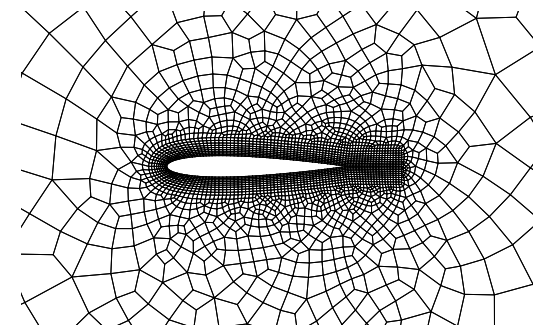

(a) Q2 quadrilateral grid, 3,466 quadrilateral cells, 14,040 nodes

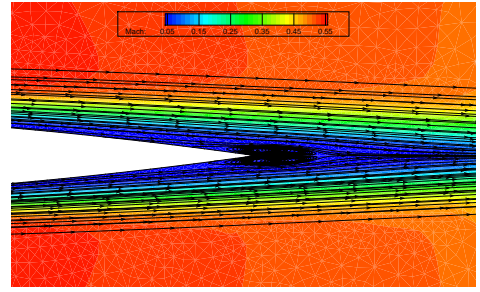

(b) Mach number contours with streamlines, 36 contours from 0.05 to 0.55 , DDG (IC) (P2) method

Figure 4: Grids and Mach number contours for laminar flow over a NACA0012 airfoil.

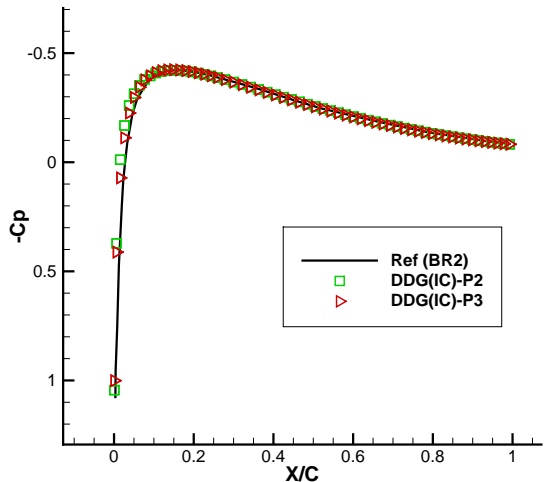

(a) Pressure coefficient

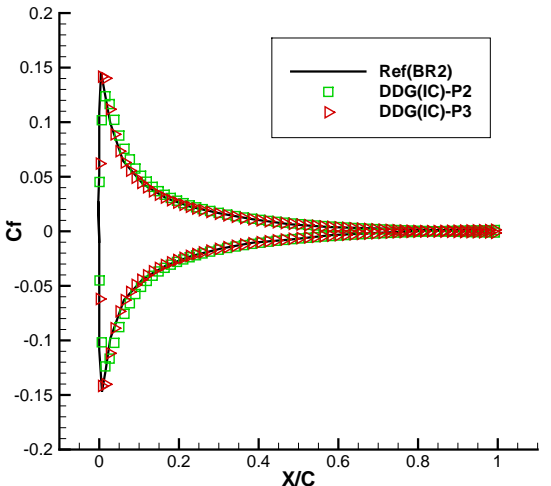

(b) Skin friction coefficient

Figure 5: Plot of pressure coefficient and skin friction coefficient for laminar flow over a NACA0012 airfoil.

Fig. 7 consists of 4,434 quadrilateral elements, and 96 boundary faces on the surface of the cylinder. The cell size is $0.0336 \mathrm{D}$ at the cylinder, with the height of the first layer grid around the cylinder equal to $0.0166 \mathrm{D}$, approximately. The computed Mach contours in the flow field and the streamlines over the cylinder are shown in Fig. 8. A comprehensive comparison of separation angle $\theta_{s}$, wake length $L_{w} / D$, and drag coefficient $C d$ is presented in Table 6 . One can observe that the results obtained by the newly developed DDG (IC) method are well within the range of the reference solutions.

\section{Conclusions}

In this paper, we have developed and investigated a new DDG method with interface correction (DDG (IC)) as the discretization of viscous and heat fluxes for the compressible Navier-Stokes equations on unstructured grids. Compared to the original DDG method, 


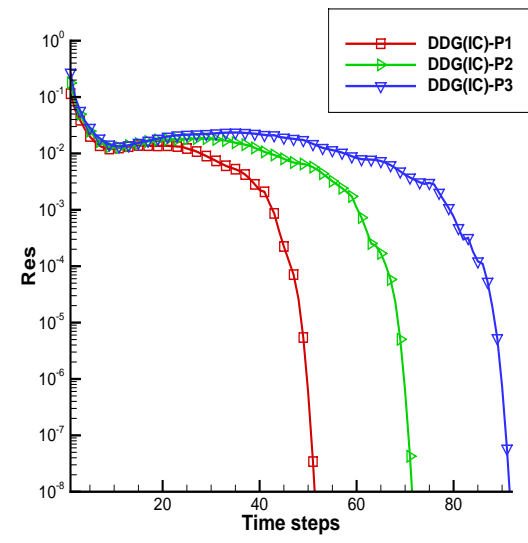

(a) Residual vs time steps

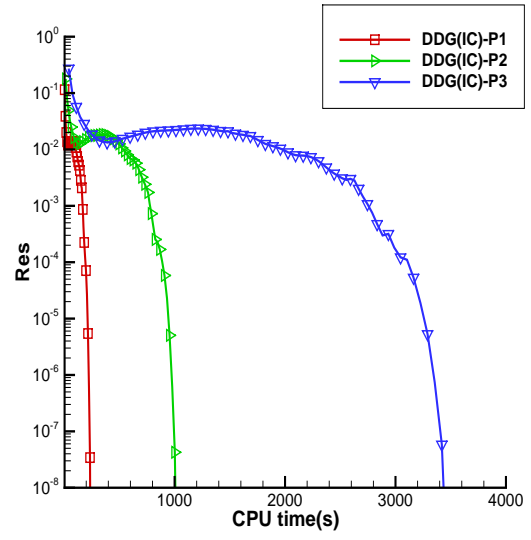

(b) Residual vs CPU time(s)

Figure 6: Convergence history of laminar flow over a NACA0012 airfoil.

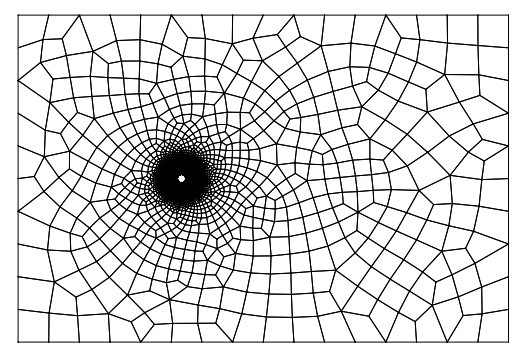

(a) Q2 quadrilateral grid, 4,434 cells, 17,884 nodes

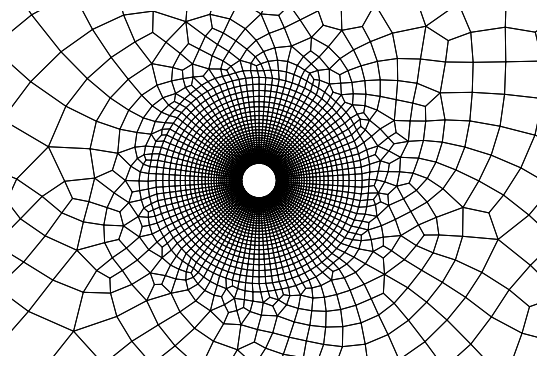

(b) Zoom in near the cylinder

Figure 7: Hybrid structured and unstructured quadrilateral grid for steady flow past a cylinder.

the DDG method with interface correction has several superior potentials. It no longer requires the jump terms of even order derivatives higher than two, meanwhile becomes more robust in delivering the designed optimal order of accuracy under demanding situations, i.e., under severely distorted grids. A number of test cases have been presented to assess the performance the DDG method with interface correction for solving the com-

Table 6: Comparison of separation angle $\theta_{S}$, wake length $L_{w} / D$, and drag coefficient $C d$ for steady flow past a cylinder.

\begin{tabular}{||c|c|c|c||}
\hline Method & $\theta_{s}$ & $L_{w} / D$ & $C d$ \\
\hline DDG (IC) (P1) & $123.6^{\circ}$ & 2.11 & 1.57694 \\
DDG (IC) (P2) & $125.9^{\circ}$ & 2.17 & 1.56831 \\
DDG (IC) (P3) & $126.2^{\circ}$ & 2.18 & 1.56149 \\
\hline Reference [28] & $125.8^{\circ}-127.3^{\circ}$ & $2.13-2.35$ & $1.48-1.62$ \\
\hline
\end{tabular}




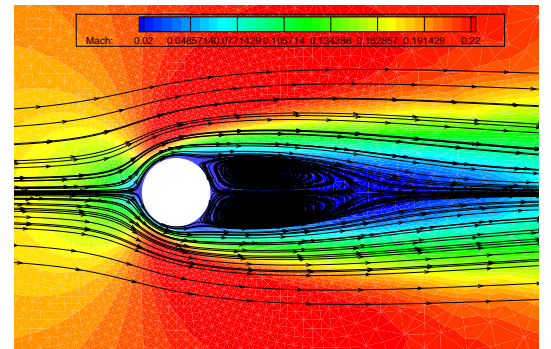

(a) 36 Mach number contours, from 0.02 to 0.22, DDG (IC) (P2) method

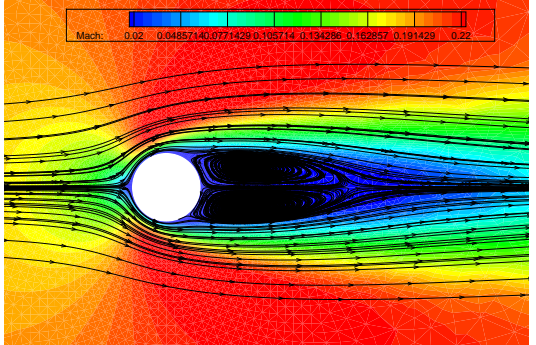

(b) 36 Mach number contours, from 0.02 to 0.22 , DDG (IC) (P3) method

Figure 8: Mach number contours and streamlines for steady flow past a cylinder.

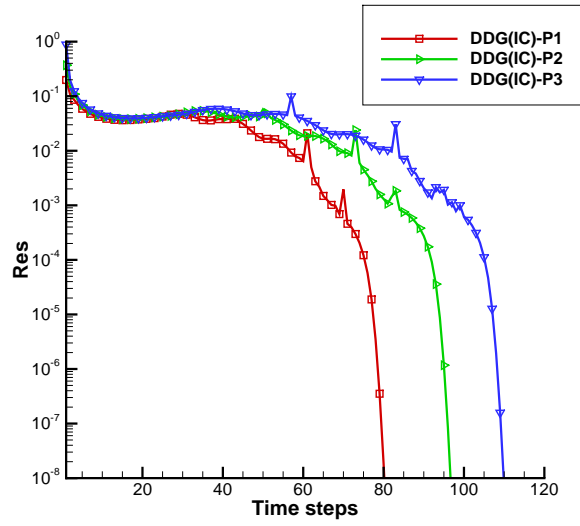

(a) Residual vs time steps

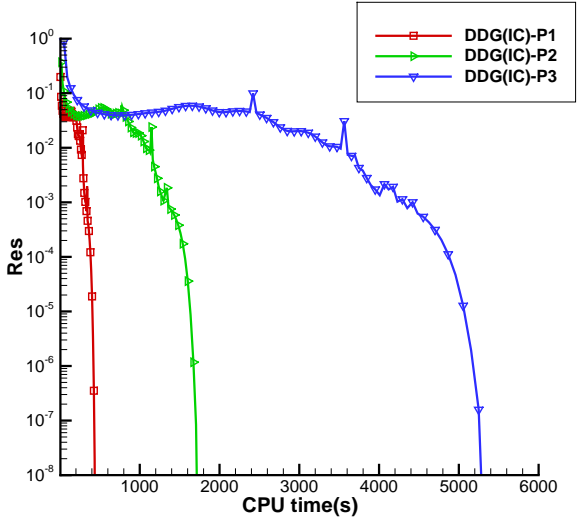

(b) Residual vs CPU time(s)

Figure 9: Convergence history of steady flow past a cylinder.

pressible Navier-Stokes equations. Numerical experiments demonstrate that DDG (IC) method can achieve the optimal order of accuracy on both uniform structured grids and nonuniform unstructured grids, which clearly indicates its potential for further applications of real engineering practices.

\section{Acknowledgements}

The authors would like to acknowledge the partial support provided by National Natural Science Foundation of China No. 10931004 and No. 9153030001, Fundamental Research of Civil Aircraft No. MJ-F-2012-04. Computations in this work have been performed based on deal.II library [32]. 


\section{References}

[1] W. H. REED AND T. R. HILL, Triangular mesh methods for the neutron transport equation, Los Alamos Scientific Laboratory Report, LA-UR-73-479, 1973.

[2] B. COCKBURN, S. HOU AND C.-W. SHU, TVD Runge-Kutta local projection discontinuous Galerkin finite element method for conservation laws IV: the multidimensional case, Math. Comput., 55 (1990), pp. 545-581.

[3] B. COCKBURN AND C.-W. SHU, The Runge-Kutta discontinuous Galerkin method for conservation laws V: multidimensional system, J. Comput. Phys., 141 (1998), pp. 199-224.

[4] H. LuO, L. Q. LuO, R. Nourgaliev, V. A. Mousseau And N. Dinh, A reconstructed discontinuous Galerkin method for the compressible Navier-Stokes equations on arbitrary grids, J. Comput. Phys., 229 (2010), pp. 6961-6978.

[5] H. LuO, L. LuO, A. Ali, R. Nourgaliev And C. CAI, A parallel, reconstructed discontinuous Galerkin method for the compressible flows on arbitrary grids, Commun. Comput. Phys., 9 (2011), pp. 363-389.

[6] B. VAN LEER AND S. NOMURA, Discontinuous Galerkin method for diffusion, AIAA-2005-5108, 2005.

[7] B. VAN LEER AND M. LO, A discontinuous Galerkin method for diffusion based on recovery, AIAA-2007-4083, 2007.

[8] H. L. LIU AND J. YAN, The direct discontinuous Galerkin (DDG) methods for diffusion problems, SIAM J. Numer. Anal., 47 (2009), pp. 675-698.

[9] H. L. LIU AND J. YAN, The direct discontinuous Galerkin (DDG) method for diffusion with interface corrections, Commun. Comput. Phys., 8 (2010), pp. 541-564.

[10] H. L. LIU, Optimal error estimates of the direct discontinuous Galerkin method for convectiondiffusion equations, Math. Comput., 84 (2015), pp. 2263-2295.

[11] W.-X. CAO, H. L. LIU AND Z.-M. ZHANG, Superconvergence of the direct discontinuous Galerkin method for convection-diffusion equations, Numer. Methods Partial Differential Equations, doi:10.1002/num.22087, 2016.

[12] C. VIDDEN AND J. YAN, A new direct discontinuous Galerkin method with symmetric structure for nonlinear diffusion equations, J. Comput. Math., 31 (2013), pp. 638-662.

[13] H. Y. HuANG, Z. CHEN, J. LI AND J. YAN, Direct discontinuous Galerkin method and its variations for second order elliptic equations, J. Sci. Comput., 70 (2017), pp. 744-765.

[14] F. BASSI AND S. REBAY, Discontinuous Galerkin solution of the Reynolds-averaged Navier-Stokes and $k-w$ turbulence model equations, Comput. Fluids, 34 (2005), pp. 507-540.

[15] B. COCKBURN AND C.-W. SHU, The local discontinuous Galerkin method for time dependent convection-diffusion systems, SIAM J. Numer. Anal., 35 (1998), pp. 2440-2463.

[16] H. LuO, J. D. BAUM AND R. LÖHNER, A discontinuous Galerkin method based on a Taylor basis for the compressible flows on arbitrary grids, J. Comput. Phys., 227 (2008), pp. 8875-8893.

[17] J. Peraire AND P. O. PERSSON, The compact discontinuous Galerkin (CDG) method for elliptic problems, SIAM J. Sci. Comput., 30 (2008), pp. 1806-1824.

[18] F. BASSI AND S. REBAY, A high-order accurate discontinuous finite element method for the numerical solution of compressible Navier-Stokes equations, J. Comput. Phys., 131 (1997), pp. 267-279.

[19] D. N. ARNOLD, F. BREZZI, B. COCKBURN AND L. D. MARINI, Unified analysis of discontinuous Galerkin methods fro elliptic problems, SIAM J. Numer. Anal., 19 (2002), pp. 742-760.

[20] J. DOUGLAS AND T. DUPONT, Interior penalty procedures for eplliptic and parabolic Galerkin methods, Computing Methods in Applied Sciences, Lecture Notes in Phys., Springer, Berlin, 58 (1976), pp. 207-216. 
[21] M. F. WHEELER, An elliptic collocation-finite element method with interior penalties, SIAM J. Numer. Anal., 15 (1978), pp. 152-161.

[22] R. HARTMANN AND P. HOUSTON, Symmetric interior penalty DG methods for the compressible Navier-Stokes equations I: method formulation, Int. J. Num. Anal. Model., 3 (2006), pp. 1-20.

[23] R. HARTMANN AND P. HOUSTON, An optimal order interior penalty discontinuous Galerkin discretization of the compressible Navier-Stokes equations, J. Comput. Phys., 227 (2008), pp. 9670 9685.

[24] N. C. Nguyen, J. Peraire And B. COCKBuRn, An implicit high-order hybridizable discontinuous Galerkin method for linear convection-diffusion equations, J. Comput. Phys., 228 (2009), pp. 3232-3254.

[25] J. Cheng, X. Q. YANG, X. D. LiU, T. G. LiU AND H. LuO, A direct discontinuous Galerkin method for the compressible Navier-Stokes equations on arbitrary grids, J. Comput. Phys., 327 (2016), pp. 484-502.

[26] J. Cheng, X. D. LiU, T. G. LiU And H. Luo, A parallel, high-order direct discontinuous Galerkin method for the Navier-Stokes equations on 3D hybrid grids, Commun. Comput. Phys., 21 (2017), pp. 1231-1257.

[27] G. GASSNER, F. LÖRCHER AND C.-D. MunZ, A contribution to the construction of diffusion fluxes for finite volume and discontinuous Galerkin schemes, J. Comput. Phys., 224 (2207), pp. 1049-1063.

[28] R. GAutier, D. Biau AND E. LAmballais, A reference solution of the flow over a circular cylinder at $R e=40$, Comput. Fluids, 75 (2013), pp. 103-111.

[29] R. KANNAN AND Z. J. WANG, The direct discontinuous Galerkin (DDG) viscous flux scheme for the high order spectral volume method, Comput. Fluids, 39 (2010), pp. 2007-2021.

[30] R. HARTMANN, Adjoint consistency analysis of discontinuous Galerkin discretizations, SIAM J. Numer. Anal., 45 (2007), pp. 2671-2698.

[31] J. L. LOU, X. D. LIU, H. LUO AND H. NisHIKAWA, Reconstructed discontinuous Galerkin methods for hyperbolic diffusion equations on unstructured grids, AIAA-2017-0310, 2017.

[32] W. BANGERTH, R. HARTMANN AND G. KANSCHAT, deal.II-A general-purpose object-oriented finite element library, ACM Trans. Math. Softw., 33 (2007), DOI 10.1145/1268776.1268779. 